\title{
Municípios do Rio Grande do Sul: Uma Caracterização Econômica por meio do Modelo de Weaver
}

\section{Municipalities of Rio Grande do Sul: An Economic Characterization Through the Weaver Model}

Guilherme Cardoso da Silva * Mauro Kumpfer Werlang **

\begin{abstract}
Resumo:
Em meio a muitas discussões, as técnicas quantitativas colaboraram para o avanço da ciência geográfica, sem deixar de lado a importância de avaliar as qualidades de determinado objeto de estudo. Neste sentido, alguns modelos foram criados para colaborar nas análises, como exemplo o Modelo de Weaver, que tem o objetivo de demonstrar qual(ais) é(são) o(s) dado(s) mais representativo(s) em um contexto predeterminado. Diante disso, o objetivo deste trabalho é apresentar uma caracterização econômica para o Estado do Rio Grande do Sul, baseado em seus municípios, utilizando o Modelo de Weaver. Para tanto, foram utilizados dados da Fundação de Economia e Estatística-FEE do ano de 2014 relativos à Agropecuária, Indústria e Serviços. Os dados foram tabulados, analisados e espacialmente relacionados com os encabeçamentos das principais atividades econômicas e às Categorias de Diversificação Funcional. Para complementar as análises, os resultados foram comparados aos dados apresentados nos trabalhos de Müller Filho (1983) e Rovani et al. (2008). Percebeu-se que as atividades mais significativas para o Rio Grande do Sul são as da classificação "13", relativa à soma Agropecuária e Serviços, com 241 ocorrências, sendo $48,5 \%$ do total, e que a categoria bifuncional foi a mais representativa, principalmente pela influência da classificação "13", totalizando 63,58\%. Conclui-se que o Modelo de Weaver é bastante pertinente na intenção de discriminar valores, dando a importância de dados específicos e possibilitando o descarte de outros menos relevantes, além de permitir a comparação com outros trabalhos que utilizaram o mesmo modelo, obedecendo essa padronização.
\end{abstract}

\begin{abstract}
:
Amid many discussions, quantitative techniques have contributed to the advancement of geographic science, without neglecting the importance of evaluating the qualities of a particular object of study. In this regard, some models were created to collaborate in the analyzes, as the Weaver Model, which aims to demonstrate which is the most representative data in a predetermined context. Faced with this, the aim of this work is to present an economic characterization for the State of Rio Grande do Sul, based on their municipalities, using the Weaver Model. In order to do so, data from the Fundação de Economia e Estatística - FEE from the year 2014 was used, relating to Agriculture, Industry and Services. Data were tabulated, analyzed and spatially related to headers of the main economic activities and the Functional Diversification Categories. To complement the analyzes, the results were compared to the data presented in the Müller Filho (1983) and Rovani et al. (2008). It was noticed that the most significant activities for Rio Grande do Sul are those of the classification "13", relating to the sum of Agriculture and Services, with 241 occurrences, being $48.5 \%$ of the total, a $\mathrm{d}$ that the bifunctional category was the most representative, mainly due to the influence of the classification " 13 ", totaling $63.58 \%$. It is concluded that the Weaver Model is quite pertinent in the intention to discriminate values, giving the importance of specific data and allowing the discard of others less relevant, besides allowing the comparison with other works that used the same model, obeying this standardization.
\end{abstract}

* Graduado em Geografia, Especialista em Geomática (PPGG-UFSM), Mestre em Geografia e doutorando pelo PPGGEO- UFSM.

** Graduado e MSc. em Geografia, Doutorado em Ciência do Solo pela UFSM. Professor Associado do Departamento de Geociências do Centro de Ciências Naturais e Exatas/CCNE da UFSM

\section{Palavras-chave:}

Técnicas Quantitativas, Modelo de Weaver, Categorias de Diversificação Funcional

Keywords:

Quantitative Techniques, Weaver Model, Functional Diversification Categories 


\section{INTRODUÇÃO}

A década de 1950 é intitulada por alguns pesquisadores como a "Revolução Quantitativa" visto que esta "revolução" teria iniciado no final dos anos 40 , início dos 50, chegando ao seu auge no período entre 1957 e 1960, quando teria acabado. Dentro deste quadro há um enorme destaque para os físicos e matemáticos, primeiramente, que colaboraram com o movimento que levou a uma revolução na geografia, e posteriormente com as ciências biológicas (BURTON, 1977; BERRY \& MARBLE, 1968).

Neste contexto, há um forte destaque para o impacto do trabalho de não-geógrafos na geografia, sendo um esforço vivido em várias disciplinas, que acabaram por trazer uma abordagem matemática. Pode-se chamar de a "matematização da geografia", com a construção e testes de muitos modelos teóricos (BERRY \& MARBLE, 1968).

A transformação que caracteriza a fase contemporânea da Ciência Geográfica ficou conhecida como Nova Geografia, e teve como característica o emprego da linguagem matemática; desenvolvimento de aporte técnico e de metodologias derivadas das ciências exatas; larga utilização de tecnologias computacionais; neutralidade científica e imparcialidade do pesquisador frente ao seu objeto e predomínio da abordagem espacial (CHRISTOFOLETTI, 1976). Neste momento a geografia sofre uma grande influência da quantificação, ficando conhecido o conceito de "Geografia Quantitativa”. Galvão e Faissol (1970) comentam que é necessário destacar que a noção de revolução quantitativa não significa usar dados quantitativos em oposição a dados não qualitativos, ou seja, não é uma disputa de importância, e sim um novo recurso que a ciência geográfica se apropria para desenvolver os seus estudos.

O Modelo de Weaver é uma técnica de fácil utilização, não se tratando de cálculos complicados $\mathrm{e}$ nem necessitando de muitos recursos (MÜLLER FILHO, 1983). Weaver (1954) formulou seu modelo estatístico baseado no cálculo da variância mínima para a identificação das combinações significativas. Neste modelo há a contraposição de duas situações, uma "ideal" ou "teórica", em que se n varia de 1 a 10, com percentagens correspondentes variando de 100 a $10 \%$, e outra situação sendo a "real", em que se utiliza a percenta- gem verdadeira.

O impulso da realização do presente trabalho se deu inspirado no artigo de Müller Filho (1983), que utilizou os municípios do Rio Grande do Sul e o Modelo de Weaver para realizar uma caracterização do espaço econômico relativo a agropecuária, indústria e serviços. Acontece que os dados já estão defasados, tanto é que na época o Estado possuía 232 municípios, sendo atualmente 497, e por mais que o trabalho realizado por Rovani et al. (2008) tenha trazido dados mais recentes, foi utilizada uma escala de microrregiões e não em municípios.

Desta forma, o objetivo do presente trabalho é apresentar uma caracterização econômica para o Estado do Rio Grande do Sul, baseado em seus municípios, utilizando o Modelo de Weaver.

\section{MATERIAIS E MÉTODOS}

Diante do objetivo de classificar economicamente todos os municípios do Estado do Rio Grande do Sul, fica-se dependente dos dados mais recentes disponíveis por órgãos nacionais ou estaduais. Frente ao exposto, os dados mais recentes encontrados, e julgados pertinentes à pesquisa, foram os disponibilizados pela Fundação de Economia e Estatística - FEE, do ano de 2014, considerando a participação na economia relativa à Agropecuária, Indústria e Serviços para os 497 municípios.

Desta forma, inicialmente foi realizada a revisão da bibliografia, principalmente a voltada às técnicas quantitativas e ao Modelo de Weaver e, posteriormente foi estabelecida a busca pela base de dados. A partir de então foi aplicado o Modelo de Weaver com, basicamente, as seguintes etapas (WEAVER, 1954):

a) tabulação inicial dos dados relativos à participação da atividade Agropecuária, Indústria e Serviços para cada um dos 497 municípios do estado do Rio Grande do Sul;

b) estabelecimento da percentagem para cada município de acordo com o valor relativo à participação da atividade, Agropecuária, Indústria e Serviços (considerada como percentegem "real" no modelo de Weaver);

c) ordenação dos dados em percentagens decrescentes;

d) cálculo das percentagens teóricas correspon- 
dentes as $\mathrm{n}$ categorias de atividade consideradas, em que $\mathrm{n}=1$, a categoria de atividade terá uma distribuição percentual teórica de $100 \% ; n=2$, as categorias de atividades terão cada um $50 \%$ da distribuição; $\mathrm{n}=3$, cada categoria de atividade corresponderá a 33,33\% da repartição;

e) cálculo da diferença entre a percentagem real e a percentagem teórica, sucessivamente para a categoria de atividade com maior percentual, em seguida, para as categorias de atividades com maiores percentagens;

f) cálculo do quadrado da diferença;

g) soma dos quadrados das diferenças constatadas, e a posterior soma sendo dividida em cada combinação progressivamente pelo número $\mathrm{n}$ de categorias que entram em cada cálculo parcial, sendo aqui estabelecida a variância estatística e então, a menor variância obtida corresponde à combinação significativa.

Para melhor compreender esta etapa, é apresentada a tabela 1, com o exemplo do município de Santa Maria, um dos 497 municípios do Rio Grande do Sul analisados.

Tabela 1- Modelo de Weaver aplicado ao município de Santa Maria para as categorias de atividades Agropecuárias (1), Indústria (2) e Serviços (3)

\begin{tabular}{|c|c|c|c|c|c|c|}
\hline categoria de atividade & 3 & 3 & 2 & 3 & 2 & 1 \\
\hline$\%$ da atividade & 52,65 & 52,65 & 39,07 & 52,65 & 39,07 & 8,26 \\
\hline$\%$ teórico & 100 & 50 & 50 & 33,33 & 33,33 & 33,33 \\
\hline diferença & 47,34 & $-2,65$ & 10,92 & $-19,32$ & $-5,74$ & 25,06 \\
\hline quadrado da diferença & 2241,69 & 7,04 & 119,27 & 373,39 & 33,04 & 628,10 \\
\hline $\begin{array}{l}\text { soma dos quadrados } \\
\text { da diferença }\end{array}$ & 2241,69 & & 6,31 & & 1034,54 & \\
\hline $\begin{array}{l}\text { soma dividida pelo } \\
\text { número de atividades }\end{array}$ & 2241,69 & & ,15 & & 344,84 & \\
\hline
\end{tabular}

Fonte: Organização dos autores

Observa-se que no município de Santa Maria, considerando-se as três categorias de atividades, a Agropecuária (1) representa 8,26\%, a Indústria (2) $39,07 \%$ e os Serviços 47,34\%. Assim, como visto na tabela 1, cada coluna corresponde a uma atividade econômica e cada linha a uma operação a ser realizada, objetiva-se então estabelecer a distância mínima, ou seja, o menor valor encontrado ao final das três combinações, sendo elas: apenas a 3; 3 e 2; 3, 2 e 1. Percebe-se que o menor valor obtido é 63,15 , ou seja, a variância mínima foi encontrada na combinação 3 e 2 , ou seja, as categorias de atividades Serviços e Indústria. Assim, de acordo com o Modelo de Weaver aplicado a essas categorias de atividades, Santa Maria é um município caracterizado por duas atividades econômicas e pode ser considerado de caráter bifuncional.

Após esta etapa, foram geradas algumas tabelas para identificar particularidades dos municípios com relação às categorias de atividades econômicas. Percebe-se 15 classificações de atividades econômicas, sendo elas: 1, 2, 3, 12, 13, 21, 23, 31, 32, 123, 132, 213, 231, 312 e 321. Foi estipulado inicialmente quantas correspondências haviam para cada classificação e assim foi gerada uma tabela de frequência individual. Em seguida foi gerada uma tabela de frequência por encabeçamento da categoria de atividade. Posteriormente, foram classificados os municípios com relação às categorias de diversificação funcional (monofuncional, bifuncional e trifuncional), sendo por fim também gerada uma tabela com as frequências para estas categorias.

Com a tabulação dos dados do trabalho concluídos, fez-se uma comparação com artigos publicados anteriormente que também fizeram uso do Modelo de Weaver, para tecer algumas considerações relativas à caracterização econômica do Estado. Os resultados estão apresentados em mapeamentos, espacializando os resultados encontrados, com a utilização de dados da base cartográfica da malha municipal do estado do Rio Grande do Sul em formato shapefile disponíveis no site do Instituto Brasileiro de Geografia e Estatística - IBGE.

\section{RESULTADOS E DISCUSSÃO}

Baseado no modelo proposto por Weaver (1954) pode-se classificar os 497 municípios do Rio Grande do Sul em 15 categorias sendo elas 1, 2, 3, 12, 13, 21, 23, 31, 32, 123, 132, 213, 231, 312 e 321. A partir de então, julgou-se necessária a análise da classificação dos municípios por encabeçamento, baseada na atividade econômica predominante. Desta forma, a tabela 2 mostra os resultados obtidos para os $497 \mathrm{mu}$ nicípios do Rio Grande do Sul de acordo com o encabeçamento das atividades econômicas.

Fica evidente o destaque da combinação "13" (1 e 3), relativos à Agropecuária e Serviços, com frequência de 241 ocorrências, sendo bastante superior a todas as outras, visto que em segundo lugar está a combinação "23" (2 e 3), Indústria e Serviços, com 41 ocorrências, um valor bastante inferior.

A predominância da combinação 13 reflete a alta 
percentagem relativa ao encabeçamento da atividade Agropecuária no Estado, totalizando 339 casos (68,2\%), seguido do encabeçamento, com 104 casos, relativo à Indústria $(20,9 \%)$ e os 54 casos dos Serviços (10,9\%). Para melhor compreender este resultado, julgou-se necessário a espacialização dos dados mostrados na figura 1.

Tabela 2 - Resultado obtido com a aplicação do Modelo de Weaver para os 497 municípios do Rio Grande do Sul. Frequência de municípios de acordo com o encabeçamento das categorias econômicas: Agropecuária, Indústria e Serviços

\begin{tabular}{l|cccccr}
\hline \multicolumn{2}{c|}{ Agropecuária } & \multicolumn{2}{c}{ Indústria } & \multicolumn{2}{c}{ Serviços } \\
\hline \multicolumn{1}{c|}{ Código } & Frequência & Código & Frequência & Código & Frequência \\
\hline 1 & 36 & 2 & 26 & 3 & 2 \\
12 & 4 & 21 & 1 & 31 & 14 \\
\hline 13 & 241 & 23 & 41 & 32 & 15 \\
123 & 19 & 213 & 13 & 312 & 10 \\
\hline 132 & 39 & 231 & 23 & 321 & 13
\end{tabular}

Fonte: Organização dos autores

É importante observar que na pesquisa de Rovani et al. (2008), o encabeçamento predominante foi o dos Serviços, com 91,4\% do total, seguido da Agropecuária, 5,7\%, e Indústria, 2,9\%, e na de Müller Filho (1983) em que se obteve a Agropecuária com 69,8\%, Indústria, 24,6\% e Comércio, 5,6\%. Percebe-se a grande discrepância entre os dados da presente pesquisa com os de Rovani et. al (2008), que se explica pela diferente escala de dados utilizada, municipal na primeira e microrregional na segunda. Já na comparação entre o presente trabalho e os dados de Müller Filho (1983), nota-se uma semelhança bastante grande, confirmando a manutenção por décadas da predominância e importância da atividade agropecuária no estado do Rio Grande do Sul. Essa comparação, em relação às percentagens, pode ser observada nos dados contidos na tabela 3 .

Tabela 3 - Comparação dos percentuais das categorias econômicas nas diferentes pesquisas consideradas

\begin{tabular}{l|ccr}
\hline \multicolumn{1}{c|}{ Autor/Atividade econômica } & Agropecuária & Indústria & \multicolumn{1}{c}{ Serviços } \\
\hline Atual pesquisa & $68,20 \%$ & $20,90 \%$ & $10,90 \%$ \\
\hline Rovani et al. (2008) & $5,70 \%$ & $2,90 \%$ & $91,40 \%$ \\
Müller Filho (1983) & $69,80 \%$ & $24,60 \%$ & $5,60 \%$ \\
\hline
\end{tabular}

Fonte: Organização dos autores.

A partir da classificação dos municípios por Categorias de Diversificação Funcional, em monofuncional, bifuncional e trifuncional, observa-se, conforme mostram os dados contidos na tabela 4, que o Rio Grande do Sul apresenta estrutura predominantemente bifuncional.

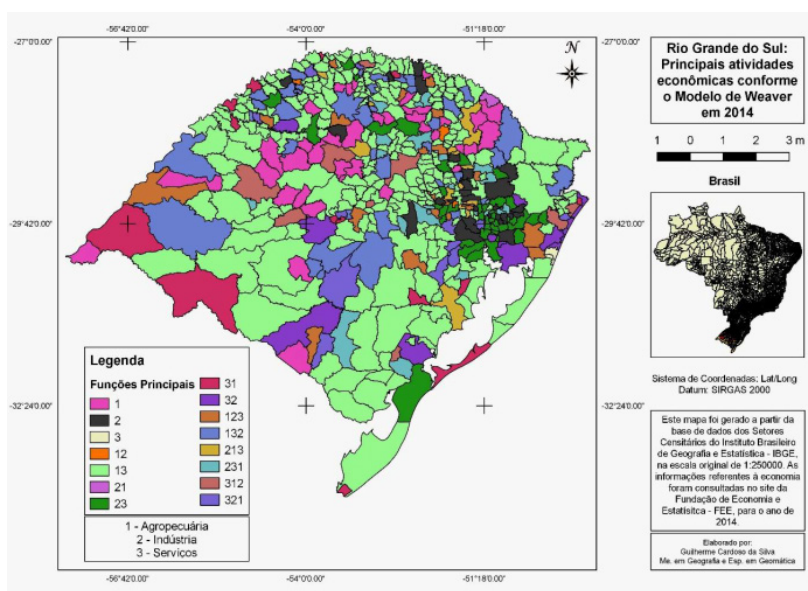

Figura 1- Mapa do Estado do Rio Grande do Sul com as categorias econômicas obtidas conforme aplicação do Modelo de Weaver. Fonte: Base cartográfica da malha municipal do estado do Rio Grande do Sul. Instituto Brasileiro de Geografia e Estatística - IBGE (2014)

A classificação por Categorias de Diversificação Funcional econômica para o Rio Grande do Sul de acordo com a aplicação do Modelo de Weaver reflete a influência da combinação Agropecuária e Serviços, que destacam a estrutura bifuncional. Ao se considerar às ocorrências também bifuncionais encabeçadas pela Indústria e pelo Comércio, obtém-se 63,58\% do Estado do Rio Grande do Sul na combinação bifuncional. A estrutura trifuncional apresenta $23,54 \%$, e por último, a estrutura monofuncional, com $12,88 \%$.

$\mathrm{Na}$ pesquisa de Rovani et al. (2008) a estrutura bifuncional também se apresentou predominante, com $54,3 \%$, seguida da trifuncional com 40\%, e monofuncional com apenas 5,7\%. Nota-se que a ordem decrescente de bifuncional, trifuncional e monofuncional permanecem apenas havendo uma proeminência para uma maior relevância da estrutura trifuncional em Rovani et al. (2008) frente ao destaque bem maior da bifuncional encontrado na presente pesquisa.

Tabela 4 - Classificação por Categorias de Diversificação Funcional econômica para o Rio Grande do Sul de acordo com a aplicação do Modelo de Weaver

\begin{tabular}{l|cccrrr}
\hline Estrutura & Agropecuária & Indústria & Serviços & Total & $\begin{array}{c}\text { \% sobre o } \\
\text { total }\end{array}$ \\
\hline Monofuncional & 36 & 26 & 2 & 64 & 12,88 \\
\hline Bifuncional & 245 & 42 & 29 & 316 & 63,58 \\
Trifuncional & 58 & 36 & 23 & 117 & 23,54 \\
\hline \\
ronte: Urganızaçao dos autores.
\end{tabular}

Fonte: Urganızaçao dos autores.

Nos resultados obtidos por Müller Filho (1983), tem-se a estrutura monofuncional com 47,8\%, a bifun- 
cional 29, $8 \%$ e a trifuncional 19,8\%. Cabe destacar que de acordo com esses dados ainda se pode analisar municípios polifuncionais, com quatro ou mais atividades predominantes. Assim, levando em consideração que esta última estrutura totalizou apenas 2,6\% do total. Portanto, em 1983 a estrutura monofuncional era dominante, em quase $50 \%$ dos municípios do Estado. Para melhor compreender estes resultados, julgou-se pertinente a elaboração de uma tabela comparativa com os resultados das três pesquisas (tabela 5) e a espacialização dos dados do presente estudo (figura 2).

Tabela 5 - Comparação dos percentuais das Categorias de Diversificação Funcional econômica nas diferentes pesquisas

\begin{tabular}{c|cccc}
\hline $\begin{array}{c}\text { Autor/Categoria } \\
\text { de } \\
\text { diversificação } \\
\text { funcional }\end{array}$ & Monofuncional & Bifuncional & Trifuncional & Polifuncional \\
\hline Atual pesquisa & $12,88 \%$ & $63,58 \%$ & $23,54 \%$ & \\
\hline $\begin{array}{c}\text { Rovani et al. } \\
(2008)\end{array}$ & $5,70 \%$ & $54,30 \%$ & $40 \%$ & \\
$\begin{array}{c}\text { Müller Filho } \\
(1983)\end{array}$ & $47,80 \%$ & $29,80 \%$ & $19,80 \%$ & $2,60 \%$ \\
\hline
\end{tabular}

Fonte: Organização dos autores

O mapa do Rio Grande do Sul, visualizado na figura 2, com as Categorias de Diversificação Funcional obtidas conforme o Modelo de Weaver, ilustra o predomínio da categoria bifuncional seguida da trifuncional e, por último a monofuncional. Observa-se que nenhuma das categorias funcionais para os municípios se apresenta concentrada e sim dispersa por todo o território gaúcho.

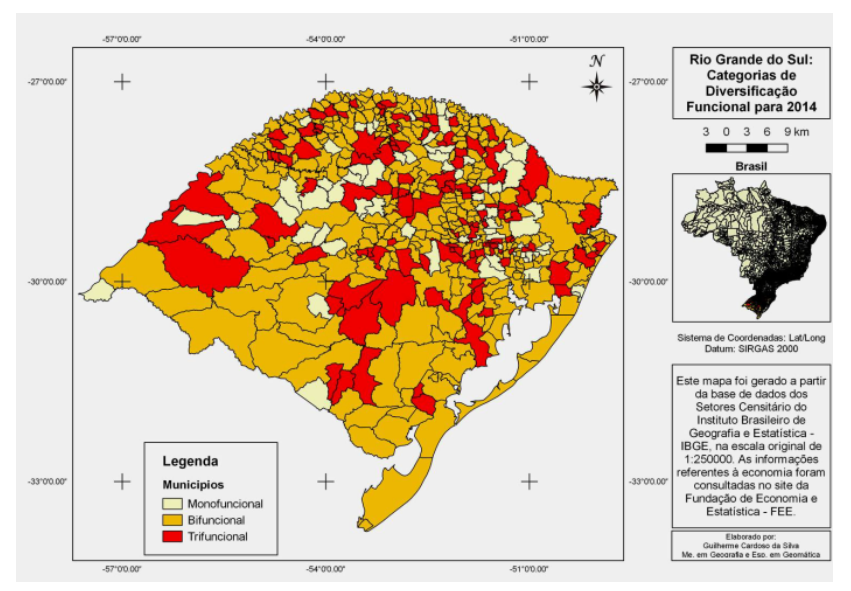

Figura 2 - Mapa do Rio Grande do Sul com as Categorias de Diversificação Funcional conforme o Modelo de Weaver

Fonte: Base cartográfica da malha municipal do estado do Rio Grande do Sul. Instituto Brasileiro de Geografia e Estatística - IBGE (2014)

\section{CONSIDERAÇÕES FINAIS}

O resultado obtido evidencia a combinação das atividades agropecuárias e serviços mostrando que os municípios do Estado do Rio Grande do Sul estão predominantemente caracterizados pela agropecuária e prestação de serviços. A agropecuária caracteriza a estrutura mais representativa entre as três consideradas no presente trabalho. Em relação às Categorias de Diversificação Funcional, observa-se o predomínio da estrutura Bifuncional, influenciada pela combinação agropecuária e serviços.

Em comparação com os trabalhos anteriores, na pesquisa de Rovani et al. (2008) a estrutura bifuncional também se apresentou predominante e, os resultados obtidos por Müller Filho (1983), mostram o predomínio da estrutura monofuncional, ou seja, conclui-se que os municípios do Rio Grande do Sul ainda mantêm sua base econômica nas atividades agropecuárias embora a categoria serviços tenha crescido em importância nas últimas décadas.

A aplicação do Modelo de Weaver permitiu a classificação por Categorias de Diversificação Funcional econômica para o Rio Grande do Sul mostrando que nenhuma delas se apresenta concentrada, indicando que as atividades econômicas ocorrem de forma dispersa em todo o Estado.

\section{REFERÊNCIAS}

BURTON, I. A revolução quantitativa e a Geografia teorética. Geografia, Rio Claro, v. 7, n. 13, 1977. p. 63-84.

BERRY, B.J.L. \& MARBLE, D. F. Spatial Analysis: a reader in statistical geography. New Jersey: Prentice Hall, 1968.

CHRISTOFOLETTI, A. As características da nova geografia. Geografia, Rio Claro. n. 1, v. 1, 1976.

FEE- Fundação de Economia e Estatística. Banco de Dados. Disponível em: <http:// fee.gov.br>. Acesso em: 27 dez. 2016.

MÜLLER FILHO, I.L. Caracterização do espaço econômico do Rio Grande do Sul (uma adaptação do modelo de Weaver). Geografia, v. 8, n. 15-16, p. 129-142, 1983.

ROVANI, F.F.M.; WERLANG, M.K.; CASSOL, R. Microrregiões geográficas do Rio Grande do Sul: uma caracterização econômica a partir do Modelo de Weaver. Disciplinarum Scientia, Santa Maria, v. 9, n. 1, p. 111-120, 2008.

WEAVER, J.C.C. Combination regions in the Middle West.

The Geographical Review, v. 44, n. 2, 1954. 
Correspondência dos autores:

Guilherme Cardoso da Silva

e-mail: gcsgeo@gmail.com

Mauro Kmpfer Werlang

e-mail:wermakwer@gmail.com

Artigo recebido em: 09/06/2017

Revisado pelo autor em: 23/01/2018

Aceito para publicação em: 10/03/2018 